\title{
How to control Pseudomonas aeruginosa-induced pneumonia and keratitis? A lesson from the amphibian skin-derived peptide Esculentin(1-21) and its diastereomer
}

\author{
Maria Luisa Mangoni ${ }^{1}$, Floriana Cappiello ${ }^{1}$, Bruno Casciaro ${ }^{1}$, Chen Chen $^{2}$, Debarun Dutta ${ }^{3}$, Alison McDermott ${ }^{4}$, \\ Mark Dp. Willcox ${ }^{3}$, Peter $\mathrm{Di}^{2}$ \\ ${ }^{1}$ Sapienza University of Rome, Italy \\ ${ }^{2}$ Pittsburgh University, United States \\ ${ }^{3}$ UNSW, Sydney, Australia \\ ${ }^{4}$ NorthumbriaUniversity Newcastle upon Tyne, United Kingdom
}

https://doi.org/10.17952/35EPS.2018.228

The alarming emergence of "superbugs" that are resistant to commercially available antibiotics represents an increasingly serious threat to global public health.

The Gram-negative bacterium Pseudomonas aeruginosais among microbial pathogens for which new antiinfective agents are urgently needed. It causes a large variety of infections including pneumonia especially in cystic fibrosis (CF) sufferers and keratitis in contact lens wearers.

Naturally occurring antimicrobial peptides (AMPs) hold promise as novel therapeutics (1). They are geneencoded molecules produced by almost all living organisms as key components of the innate immune system. Amphibian skin secretions are one of the richest sources for AMPs. We recently discovered that the frog skin-derived cationic AMP Esc(1-21) has rapid killing kinetics against both free-living and biofilm forms of $P$. aeruginosa, with membrane-perturbing activity as a plausible mode of action which was shown to limit the emergence of resistance (2). This was demonstrated by the invariant minimal inhibitory concentration(MIC) of Esc(1-21) after multiple cycles of treatmentof $P$. aeruginosa in contrast with what found for the currently-used colistin or other traditional antibiotics, e.g. ciprofloxacin, tobramycin and aztreonam, whose MICs significantly increased already after 15 cycles of treatment.

Beside displaying antimicrobial activity, Esc(1-21) can also stimulate migration of bronchial epithelial cells, stably expressing either a functional or mutated $(\Delta \mathrm{F} 508)$ form of the CF transductance regulator (CFTR). Indeed, Esc(1-21) was found to promote the closure of a pseudo-wound of epithelial cells with $500 \neq \mathrm{m}$ width, produced in a monolayer of these cells within $24 \mathrm{~h}$, at the optimal concentration of $10 \neq \mathrm{M}$ (3). This suggests that this peptide can accelerate the healing of injuries that are produced in the lung tissue, especially in CF patients, where the wound healing mechanisms are extremely slow favoring the establishment of microbial infections.

Our preliminary data have also indicated that the peptide-induced migration of bronchial epithelial cells involves the epidermal growth factor receptor (EGFR) mediated signaling pathway (3).

Among other non-antimicrobial properties, Esc(1-21) was found to display an anti-endotoxin activity, by preventing the secretion of the pro-inflammatory cytokine TNF- $\alpha$ from lipopolysaccharide (LPS)-activated macrophages (4). This is likely due to the peptide-induced disruption of LPS aggregates into smaller-sized particles. Nevertheless, we cannot ignore the limitations in developing AMPs as future therapeutics, namely: (i) the cytotoxicity; (ii) the low biostability and (iii) limited diffusion and delivery to the target site. Over the past years we discovered that substitution of two amino acids in $\operatorname{Esc}(1-21)$, i.e. Leu ${ }^{14}$ and $\operatorname{Ser}^{17}$ with the corresponding D-enantiomers (to obtain the diastereomer Esc(1-21)-1c) is sufficient to drastically reduce the cytotoxicity of the peptide against a large number of mammalian cells. Furthermore, these two $\mathrm{D}$-amino acids substitution is sufficient to significantly increase the peptide stability not only in biological fluids, but also in the presence of proteolytic enzymes that are abundant in the lungs of CF patients (e.g. elastase from human neutrophils). In addition, the presence of these two $\mathrm{D}$-amino acids can confer the peptide a higher antibiofilm activity and improved wound healing activity, with an optimal concentration of $1 \neq \mathrm{M}$ compared to $10 \neq \mathrm{M}$ for the all-L peptide (3). Importantly when tested in vivo in murine models of acute lung infection, a single intra-tracheal instillation of the diastereomer at a very low dosage $(0.1 \mathrm{mg} / \mathrm{kg})$ was found to cause 2-log reduction in the lung bacterial burden, $24 \mathrm{~h}$ after infection without provoking an inflammatory response, similarly to colistin (5). However, in contrast with Esc(1-21)-1c, colistin is active only against Gram-negative bacteria, it induces antibiotic resistance and does not have any wound healing activity.

As mentioned above, $P$. aeruginosa is also responsible for keratitis, especially in contact lenses (CLs) wearers, 
because of the ability of this pathogen to strongly adhere to the silicone hydrogel CLs forming a biofilm. Among the most feasible approaches to reduce the risk of CL-associated keratitis there is the eradication of bacterial biofilm formed on CLs and the prevention of bacterial colonization of this medical device. We recently found out that the diastereomer can rapidly kill Pseudomonas biofilm formed on both sides of CLs. Most importantly, we demonstrated that covalent binding of the peptides to silicone hydrogel soft CL e.g. etafilcon A, represents and effective strategy to achieve an antimicrobial medical device $(2 \neq \mathrm{g}$ peptide per lens). More precisely, metacrylic acid present in the lens matrix was activated by 1-Ethyl-3-(3-dimethylaminopropyl) carbodiimide and functionalized with the peptide via carbodiimide coupling. The resulting peptideimmobilized CLs were able to cause 4-log decrease in the number of bacterial cells in $20 \mathrm{~min}$. Furthermore, they were able to reduce bacterial adhesion to the lens surface. This is expected to hamper the bacterial transfer from the CL to the cornea, and therefore the establishment of infection.

Remarkably, such peptide conjugation did not make CLs toxic to mammalian cells and did not alter the lens parameters, e.g. diameter, center thickness whose values were similar to those of control lenses; and no detectable abnormalities were obtained for the peptide-conjugated CLs when examined under a Nikon profile projector.

We also proved that $\operatorname{Esc}(1-21)$ can retain its bactericidal activity in the presence of human basal tears and reduce the level of infection in murine models of keratitis after three application per day for 3 days at a concentration of $40 \neq \mathrm{M}$.

Overall, these peptides represent attractive alternatives to control Pseudomonas-associated pneumonia and keratitis (Fig.1).

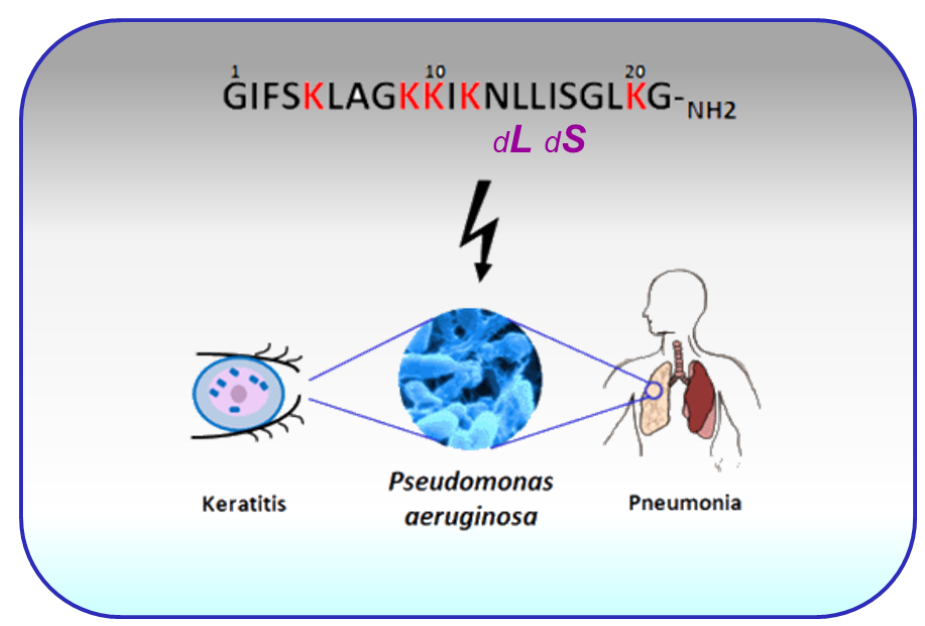

Figure 1: Schematic representation for the potential application of Esc peptides against Pseudomonas aeruginosa-induced infection. $d L$ and $d S$ indicate amino acids in the D-configuration. Basic residues are marked in red

\section{Acknowledgments}

This work was supported by grants from Sapienza University of Rome; the Italian Cystic Fibrosis Research Foundation (Project FFC\#11/2014; FFC\#15/2017); NIH (R01 HL-125128 and AI-133361), NHMRC development grant (APP107620) and an ARC discovery grant (DP160101664) from the Australian Government

\section{References}

1. Zasloff M. Nature (2002) 415: 389-3952. doi: 10.1038/415389a

2. Luca V et al. Cell Mol Life Sci. (2013) 70:2773-2786. doi: 10.1007/s00018-013-1291-7.

3. Cappiello F et al. Antimicr Agents Chemother(2016) 60:7252-7262. doi: 10.1128/AA C.00904-16

4. Di Grazia et al. Aminoacids (2015) 47:2505-19. doi:10.1007/s00726-015-2041-y

5. Chen C et al. Sci Rep. (2017) 7(1):8548. doi: 10.1038/s41598-017-08361-8. 